(c) VSP 1996.

\title{
On vector invariants of the symmetric group*
}

\author{
S. A. STEPANOV
}

Abstract - The purpose of this paper is to give a proof of the results announced by the author [7] in 1982 on the algebraic independence over a field $k$ of any non-degenerate system of $m n$ distinct basis invariants in the ring $k\left[x_{11}, \ldots, x_{1 n} ; \ldots ; x_{m 1}, \ldots, x_{m n}\right]$ with respect to the symmetric group $G=S_{n}$. The result of this paper can be extended to the case of an arbitrary finite group.

The work was partially supported by the Russian Foundation for Basic Research, Grant 94-01-01206-a.

\section{INTRODUCTION}

Let $m, n$ be positive integers, $k$ be a field of characteristic 0 and let

$$
A_{m n}=k\left[x_{11}, \ldots, x_{1 n} ; \ldots ; x_{m 1}, \ldots, x_{m n}\right]
$$

be the algebra of polynomials in $m n$ indeterminates $x_{i j}, i=1, \ldots, m, j=1, \ldots, n$. The symmetric group $G=S_{n}$ operates on the algebra $A_{m n}$ as a group of $k$-automorphisms by the rule $g x_{i j}=x_{i, g(j)}, g \in G$. Denote by $A_{m n}^{G}$ the subalgebra of invariants of the algebra $A_{m n}$ with respect to the group $G$ and define elementary symmetric polynomials $u_{r_{1}, \ldots . r_{m}} \in A_{m n}^{G}$ of vectors $\left(x_{11}, \ldots, x_{1 n}\right), \ldots,\left(x_{m 1}, \ldots, x_{m n}\right)$ by means of the formal identity

$$
\prod_{j=1}^{n}\left(1+x_{1 j} z_{1}+\ldots+x_{m j} z_{m}\right)=1+\sum_{1 \leq r_{1}+\ldots+r_{m} \leq n} u_{r_{1}, \ldots, r_{m}} z_{1}^{r_{1}} \ldots z_{m}^{r_{m}} .
$$

The polynomials $u_{r_{1}, \ldots ., r_{m}}$ form a basis of the algebra $A_{m n}^{G}$ (see [5], [1, A.IV, p. 62], [2, p.9], and [8, p. 37]). In other words, each element $F$ of the algebra $A_{m n}^{G}$ may be represented as a polynomial in $u_{r_{1}, \ldots, r_{m}}$ with coefficients from $k$. That basis contains $\left(\begin{array}{c}m+n \\ m\end{array}\right)-1$ elements connected with one another by different algebraic relations.

On the other hand by the Noether normalization theorem (see [5] and [2, p. 17]), there exist $m n$ algebraically independent basis invariants $u_{1}, \ldots, u_{m n}$ among $u_{r_{1}, \ldots, r_{m}}$ such that $A_{m n}^{G}$ is finitely generated over $k\left[u_{1}, \ldots, u_{m n}\right]$ as a module. This means that the transcendence degree of $A_{m n}^{G}$ over $k$ is equal to $m n$ (see also [3, p. 68]). Since any invariant $u \in A_{m n}^{G}$ is algebraically dependent on $u_{1}, \ldots, u_{m n}$, the set $\left\{u_{1}, \ldots, u_{m n}\right\}$ can be also considered as a basis of another type in the algebra $A_{m n}^{G}$. The theorem of Noether provides only the

${ }^{*}$ UDC 519.4. Originally published in Diskretnaya Matematika (1996) 8, No. 2 (in Russian). 
existence of such a basis. The purpose of this paper is to give an effective version of the Noether result.

We say that a system of distinct basis invariants $u_{r_{1}, \ldots, r_{m}}$ is non-degenerate if for every positive integer $\mu \leq m$ and any integral sequence $\left(i_{1}, \ldots, i_{\mu}\right), 1 \leq i_{1}<\ldots<i_{\mu} \leq m$, it contains at most $\mu n$ elements $u_{r_{1}, \ldots, r_{m}}$ with the condition that for every $i \in\left\{i_{1}, \ldots, i_{\mu}\right\}$ there exists at least one $r_{i} \geq 1$ and for any $i \notin\left\{i_{1}, \ldots, i_{\mu}\right\}$ all the corresponding $r_{i}$ are zeros. Note that any subsystem of a non-degenerate system is also non-degenerate.

Theorem 1. Any $s \leq m n$ elements $u_{r_{1}, \ldots, r_{m}}$ of a non-degenerate system of distinct basis invariants are algebraically independent over the field $k$.

In addition, this paper contains a generalization of the well-known Waring's formulae (see [1, A. IV, p. 99])

$$
\sum_{j=1}^{n} x_{j}^{\sigma}=\sum_{l_{1}+2 l_{2}+\ldots+n l_{n}=\sigma} c\left(l_{1}, \ldots, l_{n}\right) u_{1}^{l_{1}} \ldots u_{n}^{l_{n}},
$$

where $u_{1}, \ldots, u_{n}$ are the elementary symmetric polynomials of the vector $\left(x_{1}, \ldots, x_{n}\right)$,

$$
c\left(l_{1}, \ldots, l_{n}\right)=(-1)^{l_{2}+2 l_{3}+\ldots+(n-1) l_{n}} \frac{\sigma\left(l_{1}+\ldots+l_{n}-1\right) !}{l_{1} ! \ldots l_{n} !},
$$

and the sum on the right-hand side is over all non-negative integers $l_{1}, \ldots, l_{n}$ satisfying the condition $l_{1}+2 l_{2}+\ldots+n l_{n}=\sigma$, to the case of any number $m \geq 1$ of vectors $\left(x_{11}, \ldots, x_{1 n}\right), \ldots,\left(x_{m 1}, \ldots, x_{m n}\right)$.

Theorem 2. Let $\sigma, \sigma_{1}, \ldots, \sigma_{m}$ be fixed, and let $l_{1}, \ldots, l_{n} ; r_{1 \mu}, \ldots, r_{m \mu} ; s_{1 v}, \ldots, s_{m v}$ be arbitrary non-negative integers satisfying the relations

$$
\begin{array}{cc}
\sigma_{1}+\sigma_{2}+\ldots+\sigma_{n}=l_{1}+2 l_{2}+\ldots+n l_{n}=\sigma \\
r_{1 \mu}+r_{2 \mu}+\ldots+r_{m \mu}=v, & 1 \leq \mu \leq l_{v}, \\
s_{1 v}+s_{2 v}+\ldots+s_{m v}=v l_{v}, & 1 \leq v \leq n,
\end{array}
$$

and let

$$
v_{s_{1}, \ldots, s_{m v}}=\sum \prod_{\mu=1}^{l_{v}} u_{r_{\mid \mu}, \ldots, r_{u \mu \nu}},
$$

where the sum is over all $r_{11}, \ldots, r_{1 l_{v}} ; \ldots ; r_{m 1}, \ldots, r_{m l_{v}}$ such that $r_{i 1}+\ldots+r_{i l_{v}}=s_{i v}, 1 \leq i \leq m$. Then

$$
\sum_{j=1}^{n} x_{1 j}^{\sigma_{1}} \ldots x_{m j}^{\sigma_{m}}=\frac{\sigma_{1} ! \ldots \sigma_{m} !}{\sigma !} \sum c\left(l_{1}, \ldots, l_{n}\right) \sum \prod_{v=1}^{n} v_{s_{1}, \ldots, s_{m v}},
$$

where the internal sum is over all $s_{11}, \ldots, s_{1 n} ; \ldots ; s_{m 1}, \ldots, s_{m n}$ such that $s_{i 1}+\ldots+s_{i n}=$ $\sigma_{i}, 1 \leq i \leq m$, and the external sum is over all non-negative integers $l_{1}, \ldots, l_{n}$, satisfying the condition $l_{1}+2 l_{2}+\ldots+n l_{n}=\sigma$. 
These results allow us to pass from $m n$ indeterminates $x_{11}, \ldots, x_{1 n} ; \ldots ; x_{m 1}, \ldots, x_{m n}$ to $m n$ new indeterminates $u_{r_{1}, \ldots, r_{m}}$, which can be chosen among the basis invariants $u_{r_{1}, \ldots, r_{m}}$ in an arbitrary way. Note also that the results can be extended easily to the case of positive characteristic $p>c(m, n)$.

\section{NOTATION AND LEMMAS}

Let $\sigma=\left(\sigma_{11}, \ldots, \sigma_{1 n} ; \ldots ; \sigma_{m 1}, \ldots, \sigma_{m n}\right)$ be a binary sequence of length $m n$ and $j_{1}, \ldots, j_{\mu}$ be the numbers of all those subsequences $\sigma=\left(\sigma_{1 j}, \ldots, \sigma_{m j}\right), 1 \leq j \leq n$, each of which has at least one non-zero component. Put $s_{i}, 1 \leq i \leq m$, equal to the number of $j, 1 \leq j \leq n$, such that $\sigma_{i j}=1$, and define the weight $w(\sigma)$ of the sequence $\sigma$ as

$$
w(\sigma)=\sum_{i=1}^{m} \sum_{j=1}^{n} \sigma_{i j}
$$

For any subset $\left\{l_{1}, \ldots, l_{s}\right\}$ of the set $\{1,2, \ldots, n\}$ we denote by $u_{r_{1}, \ldots, r_{m}}^{\left(l_{1}, l_{s}\right)}$ the elementary symmetric polynomials of the vectors $x_{i}=\left(x_{i j}\right)_{1 \leq j \leq n, j \notin\left\{l_{1}, \ldots, l_{s}\right\}}, 1 \leq i \leq m$, and put

$$
u_{r_{1}, \ldots, r_{m}}^{\left(l_{1}, \ldots, l_{l}\right)}= \begin{cases}1 & \text { if }\left(r_{1}, \ldots, r_{m}\right)=(0, \ldots, 0) \\ 0 & \text { if } r_{i}<0 \text { at least for one } i=1,2, \ldots, m .\end{cases}
$$

Lemma 1. Let $\sigma=\left(\sigma_{11}, \ldots, \sigma_{1 n} ; \ldots ; \sigma_{m 1}, \ldots, \sigma_{m n}\right)$ be a binary sequence of length $m n$ and let $w(\sigma)=v$.

(i) If at least one subsequence $\sigma_{j}=\left(\sigma_{1 j}, \ldots, \sigma_{m j}\right), 1 \leq j \leq n$, contains at least two components $\sigma_{i, j}=1$ and $\sigma_{i, j}=1$, then

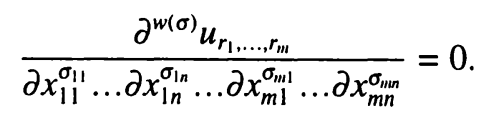

(ii) If every subsequence $\sigma_{j}=\left(\sigma_{1 j}, \ldots, \sigma_{m j}\right), 1 \leq j \leq n$, contains at most one component $\sigma_{i j}=1$, then

$$
\frac{\partial^{w(\sigma)} u_{r_{1}, \ldots, r_{m}}}{\partial x_{11}^{\sigma_{11}} \ldots \partial x_{1 n}^{\sigma_{1 n}} \ldots \partial x_{m 1}^{\sigma_{m 1}} \ldots \partial x_{m n}^{\sigma_{m n}}}=u_{r_{1}-s_{1}, \ldots, r_{m}-s_{m}}^{\left(j_{1}, \ldots, j_{v}\right)} .
$$

Proof. The first statement trivially follows from relation (1). To prove the second statement, we use induction on the weight $w(\sigma) \leq n$ of $\sigma$. For $w(\sigma)=0$ the statement is obvious. We assume that the statement is true for $w(\sigma)=v, 0 \leq v<n$, and prove its validity for $w(\sigma)=v+1 \leq n$.

Let $\sigma=\left(\sigma_{11}, \ldots, \sigma_{1 n} ; \ldots ; \sigma_{m 1}, \ldots, \sigma_{m n}\right)$ be a binary sequence with the above property and let $\left\{j_{1}, \ldots, j_{v}\right\}, w(\sigma),\left\{s_{1}, \ldots, s_{m}\right\}$ be the corresponding parameters defined by this sequence. If $w(\sigma)=v$, by the induction assumption and by definition of $u_{r_{1}-s_{1}, \ldots, r_{m}-s_{m}}^{j_{1}, j_{v}}$ we 
have

$$
\begin{aligned}
& \frac{\partial^{w(\sigma)} u_{r_{1}, \ldots, r_{m}}}{\partial x_{11}^{\sigma_{11}} \ldots \partial x_{1 n}^{\sigma_{1 n}} \ldots \partial x_{m 1}^{\sigma_{m 1}} \ldots \partial x_{m n}^{\sigma_{m n}}}=u_{r_{1}-s_{1}, \ldots, r_{m}-s_{m}}^{\left(j_{1}, \ldots, j_{v}\right)} \\
& \prod_{\substack{i=1 \\
\left\{j_{1}, \ldots, j_{v}\right\}}}^{n}\left(1+x_{1 j} z_{1}+\ldots+x_{m j} z_{m}\right)=\sum u_{r_{1}-s_{1}, \ldots, r_{m}-s_{m}}^{\left(j_{1}, \ldots j_{v}\right)} z_{1}^{r_{1}-s_{1}} \ldots . z_{m}^{r_{m}-s_{m}} \text {, }
\end{aligned}
$$

where the sum is over all $r_{1}, \ldots, r_{m}$, such that $0 \leq\left(r_{1}-s_{1}\right)+\ldots+\left(r_{m}-s_{m}\right) \leq n-v$. Denote by $j_{v+1}$ one of the numbers $j \in\{1,2, \ldots, n\}$ for which $\sigma_{j}=\left(\sigma_{1 j}, \ldots, \sigma_{m j}\right)=(0, \ldots, 0)$, and apply the operator $\partial / \partial x_{i j_{v+1}}$ to both sides of identity (4). As a result we obtain the relation

$$
\begin{aligned}
z_{i} \prod_{\substack{j=1 \\
j \in\left\{j_{1}, \ldots, j_{v+1}\right\}}}^{n}\left(1+x_{1 j} z_{1}+\ldots+x_{m j} z_{m}\right) & \\
& =\sum_{0 \leq\left(r_{1}-s_{1}\right)+\ldots+\left(r_{m}-s_{m}\right) \leq n-v}\left(\frac{\partial}{\partial x_{i j_{v+1}}} u_{r_{1}-s_{1} \ldots, r_{m}-s_{m}}^{\left(j_{1}, \ldots j_{m}\right)}\right) z_{1}^{r_{1}-s_{1}} \ldots x_{m}^{r_{m}-s_{m}} .
\end{aligned}
$$

Now we use the identity

$$
\begin{aligned}
z_{i} \prod_{\substack{j=1 \\
j \notin\left\{j_{1}, \ldots, j_{v+1}\right\}}}^{n}\left(1+x_{1 j} z_{1}+\ldots+x_{m j} z_{m}\right) \\
=z_{i}\left(\sum_{0 \leq\left(r_{1}-s_{1}\right)+\ldots+\left(r_{m}-s_{m}\right) \leq n-v-1} \sum_{0 \leq\left(r_{1}-s_{1}\right)+\ldots+\left(r_{m}-s_{m}\right) \leq n-v}^{\left.u_{r_{1}-s_{1}, \ldots, r_{m}-s_{m}}^{\left(j_{1}, \ldots j_{v+1}\right)} z_{1}^{r_{1}-s_{1}} \ldots z_{m}^{r_{m}-s_{m}}\right)}\right. \\
\quad=u_{r_{1}-s_{1}, \ldots, r_{i}-s_{i}-1, \ldots, r_{m}-s_{m}}^{\left(j_{1}, \ldots j_{v+1}\right)} z_{1}^{r_{1}-s_{1}} \ldots . z_{m}^{r_{m}-s_{m}},
\end{aligned}
$$

and then compare it with the previous relation. As a result we get

$$
\frac{\partial u_{r_{1}-s_{1}, \ldots, r_{m}-s_{m}}^{\left(j_{1}, \ldots j_{v}\right)}}{\partial x_{i j_{v+1}}}=u_{r_{1}-s_{1}, \ldots, r_{i}-s_{i}-1, \ldots, r_{m}-s_{m}}^{\left(j_{1}, \ldots j_{v+1}\right)}
$$

which implies, in view of (3), that

$$
\frac{\partial^{w(\sigma)+1} u_{r_{1}, \ldots, r_{m}}}{\partial x_{11}^{\sigma_{11}} \ldots \partial x_{1 n}^{\sigma_{l n}} \ldots \partial x_{i j_{v+1}} \ldots \partial x_{m 1}^{\sigma_{m 1}} \ldots \partial x_{m n}^{\sigma_{m n}}}=u_{r_{1}-s_{1}, \ldots, r_{i}-s_{i}-1, \ldots, r_{m}-s_{m}}^{\left(j_{1}, \ldots, j_{v+1}\right)}
$$

This proves the lemma.

Denote by $R_{m n}$ the set of all sequences $r=\left(r_{1}, \ldots, r_{m}\right)$ of non-negative integers $r_{1}, \ldots, r_{m}$, satisfying the condition $1 \leq r_{1}+\ldots+r_{m} \leq n$, and consider a subset $\mathcal{M}_{s}$ of the set $R_{m n}$ of cardinality $s$. Let us index the elements of the set $\mathcal{M}_{s}$ by the numbers $1,2, \ldots, s$, 
setting $r=r(k), 1 \leq k \leq s$, and for a given set $\mathcal{N}_{s}=\left\{\left(i_{1}, j_{1}\right), \ldots,\left(i_{s}, j_{s}\right)\right\}$ of distinct integral pairs $\left(i_{l}, j_{l}\right)$, where $1 \leq i_{l} \leq m, 1 \leq j_{l} \leq n, 1 \leq l \leq s$, let us introduce into the consideration the determinant

$$
\Delta_{s}=\operatorname{det}\left\|\frac{\partial u_{r(k)}}{\partial x_{i j}}\right\|_{(i, j) \in \mathcal{K}_{r}, 1 \leq k \leq i} .
$$

Let $\tau=\left(\tau_{11}, \ldots, \tau_{1 n} ; \ldots ; \tau_{m 1}, \ldots, \tau_{m n}\right)$ be an integral sequence of length $m n$ with nonnegative components $\tau_{i j}$ and let

$$
w(\tau)=\sum_{i=1}^{m} \sum_{j=1}^{n} \tau_{i j}
$$

be the weight of this sequence.

For each integral pair $(i, j), 1 \leq i \leq m, 1 \leq j \leq n$, we consider the set of all sequences $k_{i j}=\left(k_{i j}^{(1)}, \ldots, k_{i j}^{\left(\tau_{j j}\right)}\right)$ with mutually distinct components $k_{i j}^{(1)}, \ldots, k_{i j}^{\left(\tau_{i j}\right)} \in\{1,2, \ldots, s\}$, and put $k_{i j}=\varnothing$ for $\tau_{i j}=0$. Set

$$
\begin{aligned}
\sigma_{i j}(k) & = \begin{cases}1 & \text { if } k \in\left\{k_{i j}^{(1)}, \ldots, k_{i j}^{\left(\tau_{i j}\right)}\right\}, \\
0 & \text { if } k \notin\left\{k_{i j}^{(1)}, \ldots, k_{i j}^{\left(\tau_{i j}\right)}\right\},\end{cases} \\
\sigma(k) & =\left(\sigma_{11}(k), \ldots, \sigma_{1 n}(k) ; \ldots ; \sigma_{m 1}(k), \ldots, \sigma_{m n}(k)\right), \\
w(\sigma(k)) & =\sum_{i=1}^{m} \sum_{j=1}^{n} \sigma_{i j}(k)
\end{aligned}
$$

and denote by $\Delta_{s}\left(k_{11}, \ldots, k_{1 n} ; \ldots ; k_{m 1}, \ldots, k_{m n}\right)$ the determinant which is obtained from $\Delta_{s}$ if we replace the elements

$$
\frac{\partial u_{r(k)}}{\partial x_{i j}}, \quad(i, j) \in \mathcal{N},
$$

of the $k$ th column by the elements

$$
\frac{\partial^{w(\sigma(k))+1} u_{r(k)}}{\partial x_{11}^{\sigma_{11}(k)} \ldots \partial x_{1 n}^{\sigma_{1 n}(k)} \ldots \partial x_{m 1}^{\sigma_{m 1}(k)} \ldots \partial x_{m n}^{\sigma_{m n}(k)} \partial x_{i j}}, \quad(i, j) \in \mathcal{N}_{w} .
$$

Applying to $\Delta_{s}$ the well-known differentiation rule of determinants with functional elements and noting that

$$
\frac{\partial^{\sigma_{i i}} u_{r(k)}}{\partial x_{i j}^{\sigma_{i j}}}=0
$$

for all $k=1, \ldots, s$ and for all $(i, j), 1 \leq i \leq m, 1 \leq j \leq n$, such that $\sigma_{i j}>1$, we obtain the following result.

Lemma 2. For a given integral sequence $\tau=\left(\tau_{11}, \ldots, \tau_{1 n} ; \ldots ; \tau_{m 1}, \ldots, \tau_{m n}\right)$ with nonnegative components,

$$
\frac{\partial^{w(\tau)} \Delta_{s}}{\partial x_{11}^{\tau_{11}} \ldots \partial x_{1 n}^{\tau_{1 n}} \ldots \partial x_{m 1}^{\tau_{m 1}} \ldots \partial x_{m n}^{\tau_{m n}}}=\sum_{\left(k_{11}, \ldots, k_{m n}\right)} \Delta_{s}\left(k_{11}, \ldots, k_{1 n} ; \ldots ; k_{m 1}, \ldots, k_{m n}\right) .
$$


For a given sequence

$$
\mathcal{M}_{s}=\left\{r(k) \in R_{m n} \mid 1 \leq k \leq s\right\}, \quad 1 \leq s \leq m n,
$$

let us consider the corresponding system

$$
U_{s}=\left\{u_{r(k)} \mid r(k) \in \mathcal{M}_{s}, 1 \leq k \leq s\right\}
$$

of vector invariants $u_{r(k)}$ and denote by

$$
J_{s}=\left\|\frac{\partial u_{r(k)}}{\partial x_{i j}}\right\|_{1 \leq i \leq m, 1 \leq j \leq n, 1 \leq k \leq s}
$$

the Jacobian of the system $U_{s}$.

Lemma 3. For a given non-degenerate system

$$
U_{s}=\left\{u_{r(k)} \mid r(k) \in \mathcal{M}_{s}, 1 \leq k \leq s\right\}, \quad 1 \leq s \leq m n,
$$

of distinct vector invariants $u_{r}$, it is possible to find an integral sequence $\tau=$ $\left(\tau_{11}, \ldots, \tau_{1 n} ; \ldots ; \tau_{m 1}, \ldots, \tau_{m n}\right), 1 \leq \tau_{i j} \leq s$, and a set $\mathcal{N}_{s}=\left\{\left(i_{1}, j_{1}\right), \ldots,\left(i_{s}, j_{s}\right)\right\}$ of distinct integral pairs $\left(i_{l}, j_{l}\right), 1 \leq i_{l} \leq m, 1 \leq j_{l} \leq n, 1 \leq l \leq s$, such that

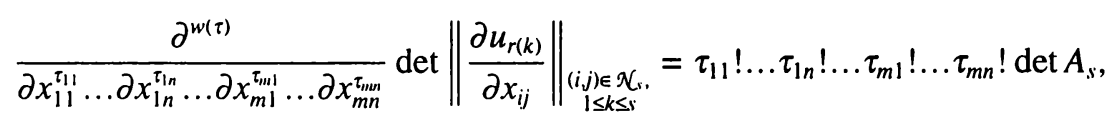

where $A_{s}$ is a binary $s \times s$ matrix of the rank $s$, which may be reduced by elementary row operations to the unit $s \times s$ matrix

$$
I_{s}=\left\|\begin{array}{ll}
1 & 0 \\
0 & 1
\end{array}\right\|
$$

Proof. We prove the lemma by induction on $s, 1 \leq s \leq m n$. Let $u_{r}=u_{r_{1} \ldots . r_{m}}$ be an arbitrary elementary symmetric polynomial in $m$ vector variables

$$
\left(x_{11}, \ldots, x_{1 n}\right), \ldots,\left(x_{m 1}, \ldots, x_{m n}\right)
$$

and let $w(r)=r_{1}+\ldots+r_{m}$ be the weight of the sequence $r=\left(r_{1}, \ldots, r_{m}\right)$.

By Lemma 1 we have

$$
\frac{\partial^{w(r)} u_{r}}{\partial x_{11} \ldots \partial x_{1 r_{1}} \partial x_{2, r_{1}+1} \ldots \partial x_{2, r_{1}+r_{2}} \ldots \partial x_{m, r_{1}+\ldots+r_{m-1}+1} \ldots \partial x_{m, r_{1}+\ldots+r_{m}}}=u_{0, \ldots, 0}^{\left(l_{1} \ldots, l_{l}\right)}=1,
$$

and hence for $s=1$ the assertion is true. 
We assume that the assertion holds for $s-1 \geq 1$ and prove its validity for $s \geq 2$. Let $\mathcal{M}_{s}=\left\{r(k) \in R_{m n} \mid 1 \leq k \leq s\right\}$ be an ordered set of cardinality $s$, consisting of elements $r=\left(r_{1}, \ldots, r_{m}\right) \in R_{m n}$, let $U_{s}=\left\{u_{r(k)} \mid r(k) \in \mathcal{M}_{s}\right\}$ be the corresponding non-degenerate system of distinct vector invariants $u_{r(k)}, 1 \leq k \leq s$, and let

$$
J_{s}=\left\|\frac{\partial u_{r(k)}}{\partial x_{i j}}\right\|_{1 \leq i \leq m, 1 \leq j \leq n, 1 \leq k \leq s}
$$

be the Jacobian of the system $U_{s}$. We assume that the set $\mathcal{M}_{s}$ is ordered in such a way that $w(r(1)) \leq w(r(2)) \leq \ldots \leq w(r(s))$. The case where all elements of the system $\mathcal{M}_{s}$ have weight 1 is trivial, hence without loss of generality we can assume that at least one sequence $r(k)=\left(r_{1}(k), \ldots, r_{m}(k)\right), 1 \leq k \leq s$, has weight $w(r(k)) \geq 2$. Thus we can assume that $v=w(r(s)) \geq 2$.

By the induction assumption we can find an integral sequence

$$
\tau^{\prime}=\left(\tau_{11}^{\prime}, \ldots, \tau_{1 n}^{\prime} ; \ldots ; \tau_{m 1}^{\prime}, \ldots, \tau_{m n}^{\prime}\right), \quad 1 \leq \tau_{i j}^{\prime} \leq s-1,
$$

and an $(s-1) \times(s-1)$ matrix

$$
H_{s-1}^{\prime}=\left\|\frac{\partial u_{r(k)}}{\partial x_{i j}}\right\|_{(i, j) \in \mathcal{N}_{s-1}^{\prime}, 1 \leq k \leq s-1}, \quad \mathcal{N}_{s-1}^{\prime}=\left\{\left(i_{1}, j_{1}\right), \ldots,\left(i_{s-1}, j_{s-1}\right)\right\},
$$

consisting of the first $s-1$ columns and $s-1$ rows of the Jacobian $J_{s}$, such that the determinant $\Delta_{s-1}^{\prime}=\operatorname{det} H_{s-1}^{\prime}$ of the matrix $H_{s-1}^{\prime}$ satisfies the relation

$$
\frac{\partial^{w\left(\tau^{\prime}\right)} \Delta_{s-1}^{\prime}}{\partial x_{11}^{\tau_{11}^{\prime}} \ldots \partial x_{1 n}^{\tau_{1 n}^{\prime}} \ldots \partial x_{m 1}^{\tau_{m 1}^{\prime}} \ldots \partial x_{m n}^{\tau_{m n}^{\prime}}}=\tau_{11}^{\prime} ! \ldots \tau_{1 n}^{\prime} ! \ldots \tau_{m 1}^{\prime} ! \ldots \tau_{m n}^{\prime} ! \operatorname{det} A_{s-1}^{\prime},
$$

where $A_{s-1}^{\prime}$ is a non-singular binary $(s-1) \times(s-1)$ matrix, which may be reduced by elementary row operations to the unit matrix $I_{s-1}$.

Now we fix the set $\mathcal{N}_{s-1}^{\prime}=\left\{\left(i_{1}, j_{1}\right), \ldots,\left(i_{s-1}, j_{s-1}\right)\right\}$ and the sequence $\tau^{\prime}=$ $\left(\tau_{11}^{\prime}, \ldots, \tau_{1 n}^{\prime} ; \ldots ; \tau_{m 1}^{\prime}, \ldots, \tau_{m n}^{\prime}\right)$ with the above mentioned properties, and show that there exists an integral pair $\left(i_{s}, j_{s}\right), 1 \leq i_{s} \leq m, 1 \leq j_{s} \leq n$, and an integral sequence $\tau=\left(\tau_{11}, \ldots, \tau_{1 n} ; \ldots ; \tau_{m 1}, \ldots, \tau_{m n}\right), 1 \leq \tau_{i j} \leq s$, such that equality (5) is valid for the determinant

$$
\Delta_{s}=\operatorname{det}\left\|\frac{\partial u_{r(k)}}{\partial x_{i j}}\right\|_{(i, j) \in \mathcal{K}_{(, 1}, 1 \leq k \leq s} \quad \mathcal{N}_{s}=\left\{\left(i_{1}, j_{1}\right), \ldots,\left(i_{s}, j_{s}\right)\right\} .
$$

Next we describe in detail the induction process necessary to construct the required pair $\left(i_{s}, j_{s}\right)$ and the required sequence $\tau=\left(\tau_{11}, \ldots, \tau_{1 n} ; \ldots ; \tau_{m 1}, \ldots, \tau_{m n}\right)$. Let

$$
\mathcal{N}_{s-1}^{\prime}=\left\{\left(i_{1}, j_{1}\right), \ldots,\left(i_{s-1}, j_{s-1}\right)\right\}, \quad \tau^{\prime}=\left(\tau_{11}^{\prime}, \ldots, \tau_{1 n}^{\prime} ; \ldots ; \tau_{m 1}^{\prime}, \ldots, \tau_{m n}^{\prime}\right)
$$

be such that the determinant $\Delta_{s-1}^{\prime}$ of matrix (6) satisfies relation (7). Let

$$
\sigma=\left(\sigma_{11}, \ldots, \sigma_{1 n} ; \ldots ; \sigma_{m 1}, \ldots, \sigma_{m n}\right)
$$


be a binary sequence with parameters

$$
v=w(\sigma)=w(r(s))=r_{1}(s)+\ldots+r_{m}(s), \quad\left(s_{1}, \ldots s_{m}\right)=\left(r_{1}(s), \ldots, r_{m}(s)\right),
$$

which has the property that any its subsequences of the form $\sigma_{\rho}=\left(\sigma_{1 \rho}, \ldots, \sigma_{m \rho}\right), 1 \leq \rho \leq n$, contains at most one component $\sigma_{i \rho}=1$. Let $\rho_{1}, \ldots, \rho_{v}$ be the numbers of all those sequences $\sigma_{\rho}=\left(\sigma_{1 \rho}, \ldots, \sigma_{m \rho}\right), 1 \leq \rho \leq n$, which contain exactly one component $\sigma_{i \rho}=1$ and assume that $j_{s} \in\left\{\rho_{1}, \ldots, \rho_{s}\right\}$. Then by Lemma 1 we have

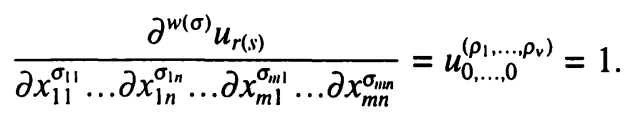

Denote by $\omega=\left(\omega_{11}, \ldots, \omega_{1 n} ; \ldots ; \omega_{m 1}, \ldots, \omega_{m n}\right)$ the binary sequence which is obtained from $\sigma=\left(\sigma_{11}, \ldots, \sigma_{1 n} ; \ldots ; \sigma_{m 1}, \ldots, \sigma_{m n}\right)$ if we replace a subsequence $\sigma_{j^{\prime}}=(0, \ldots, 0,1,0, \ldots, 0)$ beginning with $i^{\prime}$ zeros by the zero sequence $(0, \ldots, 0)$. There are

$$
L_{s}=\frac{n !}{r_{1}(s) ! \ldots r_{m}(s) !\left(n-r_{1}(s)-\ldots-r_{m}(s)\right) !}
$$

different sequences $\sigma=\left(\sigma_{11}, \ldots, \sigma_{1 n} ; \ldots ; \sigma_{m 1}, \ldots, \sigma_{m n}\right)$, satisfying relation (8), and these sequences generate

$$
M_{s}=\sum_{i=1}^{m} \frac{n !}{r_{1}(s) ! \ldots\left(r_{i}(s)-1\right) ! \ldots r_{m}(s) !\left(n-r_{1}(s)-\ldots-r_{m}(s)+1\right) !}
$$

binary sequences $\alpha=\left(\alpha_{11}, \ldots, \alpha_{1 n} ; \ldots ; \alpha_{m 1}, \ldots, \alpha_{m n}\right)$, with components

$$
\alpha_{i j}=\frac{\partial}{\partial x_{i j}}\left(\frac{\partial^{w(\omega)} u_{r(s)}}{\partial x_{11}^{\omega_{11}} \ldots \partial x_{1 n}^{\omega_{1 n}} \ldots \partial x_{m 1}^{\omega_{m 1}} \ldots \partial x_{m n}^{\omega_{i n}}}\right)
$$

which corresponds to all possible sequences $\sigma=\left(\sigma_{11}, \ldots, \sigma_{1 n} ; \ldots ; \sigma_{m 1}, \ldots, \sigma_{m n}\right)$. The sequences $\alpha$ are not necessarily distinct, but for any given $i, 1 \leq i \leq m$, they do contain exactly

$$
N_{s}=\left(\begin{array}{c}
n \\
r_{1}(s)+\ldots+r_{m}(s)-1
\end{array}\right)=\left(\begin{array}{c}
n \\
v-1
\end{array}\right)
$$

distinct sequences of the form $\alpha=\left(0, \ldots, 0 ; \ldots ; \alpha_{i 1}, \ldots, \alpha_{i n} ; \ldots ; 0, \ldots, 0\right)$, where each sequence $\alpha_{i}=\left(\alpha_{i 1}, \ldots, \alpha_{i n}\right)$ has exactly $r_{1}(s)+\ldots+r_{m}(s)-1$ zeros and $n-r_{1}(s)-\ldots-r_{m}(s)+1$ units. This provides a lot of essentially different candidates for the last column of the matrix $A_{s}$ which correspond to various integral sequences $\tau=\left(\tau_{11}, \ldots, \tau_{1 n} ; \ldots ; \tau_{m 1}, \ldots, \tau_{m n}\right)$ of the form

$$
\tau=\left(\tau_{11}^{\prime}+\omega_{11}, \ldots, \tau_{1 n}^{\prime}+\omega_{1 n} ; \ldots ; \tau_{m 1}^{\prime}+\omega_{m 1}, \ldots, \tau_{m n}^{\prime}+\omega_{m n}\right) .
$$

We will use this fact later on, and now we establish the validity of relation (5). Namely, we prove by induction on $s$ that for any given integral pair $\left(i_{s}, j_{s}\right) \notin \mathcal{N}_{i-1}^{\prime}, 1 \leq$ 
$i_{s} \leq m, 1 \leq j_{s} \leq n$, and any given integral sequence $\tau=\left(\tau_{11}, \ldots, \tau_{1 n} ; \ldots ; \tau_{m 1}, \ldots, \tau_{m n}\right)$ of form (9) relation (5) holds with a unique binary $s \times s$ matrix $A_{s}$. The matrix $A_{s}$ can be constructed as follows. Expanding the determinant $\Delta_{s}$ according to the last column, we obtain

$$
\Delta_{s}=\sum_{(i, j) \in \mathcal{K}_{r,},(i, j)=\left(i, j_{l}\right)}(-1)^{s+l} \frac{\partial u_{r(s)}}{\partial x_{i j}} \Delta_{i, j, s-1}
$$

By the induction assumption we have

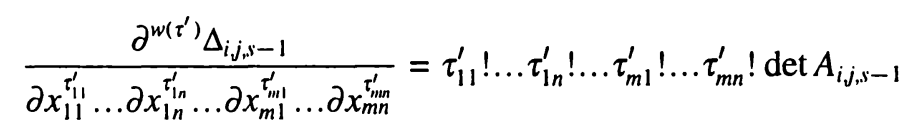

with a unique binary $(s-1) \times(s-1)$ matrix $A_{i j, s-1}$, and according to the well-known Leibniz formula we find that

$$
\begin{aligned}
& \frac{\partial^{w(\tau)} \Delta_{s}}{\partial x_{11}^{\tau_{11}} \ldots \partial x_{1 n}^{\tau_{1 n}} \ldots \partial x_{m 1}^{\tau_{m 1}} \ldots \partial x_{m n}^{\tau_{m n}}} \\
= & \sum_{(i, j) \in \mathcal{K}\left(\mathcal{K}_{r,}(i, j)=(i, j, j)\right.}(-1)^{s+l} \sum_{v_{11}=0}^{\tau_{11}} \ldots \sum_{v_{m n}=0}^{\tau_{m n}}\left(\begin{array}{c}
\tau_{11} \\
v_{11}
\end{array}\right) \ldots\left(\begin{array}{c}
\tau_{m n} \\
v_{m n}
\end{array}\right) \frac{\partial^{w(v)+1} u_{r(s)}}{\partial x_{i j} \partial x_{11}^{v_{11}} \ldots \partial x_{m n}^{v_{m n}}} \frac{\partial^{w(\tau)-w(v)-1} \Delta_{i, j, s}}{\partial x_{11}^{\tau_{11}-v_{11}} \ldots \partial x_{m n}^{\tau_{m n}-v_{m n}}} \\
= & \sum_{(i, j) \in \mathcal{K}\left(\mathcal{K}_{r,},(i, j)=(i, j l)\right.}(-1)^{s+l}\left(\begin{array}{c}
\tau_{11} \\
\omega_{11}
\end{array}\right) \ldots\left(\begin{array}{c}
\tau_{m n} \\
\omega_{m n}
\end{array}\right) \quad \omega_{11} ! \ldots \omega_{m n} ! \quad \tau_{11}^{\prime} ! \ldots \tau_{m n}^{\prime} ! \operatorname{det} A_{i, j, s-1} \\
= & \tau_{11} ! \ldots \tau_{1 n} ! \ldots \tau_{m 1} ! \ldots \tau_{m n} ! \operatorname{det} A_{s} .
\end{aligned}
$$

Further we show that for any integral pair $\left(i_{s}, j_{s}\right) \notin \mathcal{N}_{w-1}^{\prime}, 1 \leq i_{s} \leq m, 1 \leq j_{s} \leq n$, it is possible to choose an integral sequence $\tau=\left(\tau_{11}, \ldots, \tau_{1 n} ; \ldots ; \tau_{m 1}, \ldots, \tau_{m n}\right)$ of form (9) satisfying the condition that the last column of the corresponding matrix $A_{s}$ in equality (5) differs from all its other columns. For any given $l, 1 \leq l \leq s$, we denote by $n_{l}$ the number of positions $\left(i_{l}, j\right), 1 \leq j \leq n$, where the last column of the matrix

$$
H_{s}=\left\|\frac{\partial u_{r(k)}}{\partial x_{i j}}\right\|_{(i, j) \in \mathcal{N}, 1 \leq k \leq s}
$$

has exactly $n_{l}$ non-zero entries, and set $p_{s}=n_{1}+\ldots+n_{s}$. If $p_{s}=0$, then the last column of the matrix $A_{s}$ consists only of zero elements, hence it differs from all other columns of $A_{s}$. Now we assume that $p_{s} \geq 1$. It follows from the previously mentioned arguments that for any $l=1, \ldots, s$ such that $r_{i}(s) \geq 1$ with $i=i_{l}$ there is exactly $N_{s}=\left(\begin{array}{c}n \\ v-1\end{array}\right)$ distinct binary sequences $\alpha_{i}=\left(\alpha_{i 1}, \ldots, \alpha_{i n}\right)$, containing $v-1$ zeros and $n-v+1$ units. If we remove all entries of the vector $\alpha_{i}$ situated at any $n-n_{l}$ positions, then we obtain exactly

$$
q_{l}=\sum_{\sigma=v_{0}-1-\left(n-n_{l}\right)}^{v_{0}-1}\left(\begin{array}{l}
n_{l} \\
\sigma
\end{array}\right)
$$

distinct binary sequences of length $n_{l}$, where $v_{0}=\min (v, n-v+2)$ and $\left(\begin{array}{c}n_{l} \\ \sigma\end{array}\right)=0$ for $\sigma>n_{l}$ or $\sigma<0$. 
Assume that $n_{l} \geq 1$ for some $l=1,2, \ldots, s$ and consider the $n_{l}$ rows of the matrix $A_{s}$ indexed by integral pairs $\left(i_{l}, j\right) \in \mathcal{N}_{*}$ with given $i_{l}$. Let $k_{1}, \ldots, k_{\mu}, 1 \leq k_{1}<\ldots<k_{\mu}<s$, be the numbers of all those columns of $A_{s}$ which intersect the considered rows by non-zero binary vectors of length $n_{l}$, say $v_{k_{1}}, \ldots, v_{k_{\mu}}$. It follows from the structure of the matrix $A_{s}$ that all remaining entries of the corresponding columns, situated outside of the given $n_{l}$ positions, are zeros. Since the matrix $A_{s-1}^{\prime}$ is non-singular and the system $U_{s}$ is non-degenerate, we find that $\mu \leq \lambda_{l}=\min \left(n_{l}, n-1\right)$ and, moreover, that the vectors $v_{k_{1}}, \ldots, v_{k_{\mu}}$ are distinct. Thus we have $q_{l}$ different possibilities to choose the last column of the matrix $A_{s}$ with the condition that all their entries situated outside of the given $n_{l}$ positions are zeros, and only $\mu \leq \lambda_{l}$ different possibilities for all other columns with the same property. Since $q_{l}>\lambda_{l}$ for any $n_{l} \geq 1$, we can choose the last column in such a way that it differs from all other columns of the matrix $A_{s}$. Moreover, if $n_{l}>1$, we can choose the last column with the above property as a non-zero vector. Indeed, if $v_{0}=2$, then the set $\left\{v_{k_{1}}, \ldots, v_{k_{\mu}}\right\}$ contains at most one vector of weight 1 or $n_{l}-1$, and we have $n_{l}>1$ possibilities to choose the last column of $A_{s}$ as a vector of the same weight. Now, if $v_{0}>2$, we have $q_{l}>\lambda_{l}+1$, and again we can choose the last column of $A_{s}$ as a non-zero vector.

Now we fix an integral sequence $\tau=\left(\tau_{11}, \ldots, \tau_{1 n} ; \ldots ; \tau_{m 1}, \ldots, \tau_{m n}\right)$ satisfying the just described property and find an integral pair $\left(i_{s}, j_{s}\right) \notin \mathcal{N}_{s-1}^{\prime}, 1 \leq i_{s} \leq m, 1 \leq j_{s} \leq n$, which provides the condition that the matrix $A_{s}$ in (5) can be reduced to the unit matrix $I_{s}$. Set

$$
\mathcal{N}_{i-1}^{\prime \prime}=\mathcal{N}_{i} \backslash\left\{\left(i_{1}, j_{1}\right)\right\}
$$

and consider the matrix

$$
H_{s-1}^{\prime \prime}=\left\|\frac{\partial u_{r(k)}}{\partial x_{i j}}\right\|_{(i, j) \in \mathcal{K}_{s-1}^{\prime \prime}, 2 \leq k \leq s} .
$$

By the induction assumption we can find an integral pair $\left(i_{s}, j_{s}\right)$ and an integral sequence $\tau^{\prime \prime}=\left(\tau_{11}^{\prime \prime}, \ldots, \tau_{1 n}^{\prime \prime} ; \ldots ; \tau_{m 1}^{\prime \prime}, \ldots, \tau_{m n}^{\prime \prime}\right), 1 \leq \tau_{i j}^{\prime \prime} \leq \tau_{i j}$, such that the determinant $\Delta_{s-1}^{\prime \prime}=\operatorname{det} H_{s-1}^{\prime \prime}$ satisfies the relation

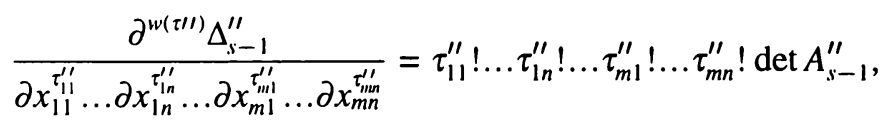

where $A_{s-1}^{\prime \prime}$ is a non-singular binary $(s-1) \times(s-1)$ matrix, which can be reduced by elementary row operations to the diagonal matrix $I_{s-1}$.

Now we fix the integral sequence $\tau^{\prime \prime}=\left(\tau_{11}^{\prime \prime}, \ldots, \tau_{1 n}^{\prime \prime} ; \ldots ; \tau_{m 1}^{\prime \prime}, \ldots, \tau_{m n}^{\prime \prime}\right)$ and the integral pair $\left(i_{s}, j_{s}\right)$ which satisfy $(10)$, and show that under the above choice of $\left(i_{s}, j_{s}\right)$ and $\tau=\left(\tau_{11}, \ldots, \tau_{1 n} ; \ldots ; \tau_{m 1}, \ldots, \tau_{m n}\right)$ the matrix $A_{s}$ in equality (5) possesses all the properties stated in the lemma. Indeed, it follows from Lemma 2 and relations (8), (9) that

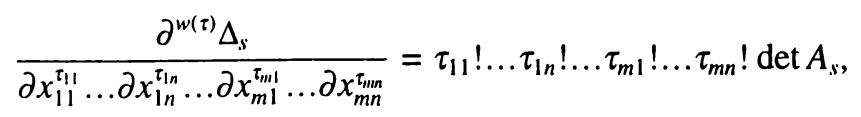

where

$$
A_{s}=\left\|a_{i j}\right\|_{1 \leq i, j \leq i-1}
$$


is a binary $s \times s$ matrix containing $(s-1) \times(s-1)$ submatrices

$$
A_{s-1}^{\prime}=\left\|a_{i j}\right\|_{1 \leq i j \leq i-1}, \quad A_{s-1}^{\prime \prime}=\left\|a_{i j}\right\|_{2 \leq i j \leq s} .
$$

Since the matrices $A_{s-1}^{\prime}$ and $A_{s-1}^{\prime \prime}$ may be transformed by elementary row operations to the unit matrix $I_{s-1}$, as a result the matrix $A_{s}$ may be reduced by the similar operations to the form

$$
\bar{A}_{s}=\left\|\begin{array}{cccc}
1 & 0 & 0 & c_{1, s} \\
0 & 1 & 0 & 0 \\
\vdots & \vdots & \vdots & \vdots \\
c_{s 1} & 0 & 0 & 1
\end{array}\right\| .
$$

Because all the columns of the matrix $A_{s}$ are distinct, all the columns of the matrix $\bar{A}_{s}$ are distinct as well. In that case $c_{1 s} c_{s 1}=0$, and hence the matrix $A_{s}$ is equivalent to the unit matrix $I_{s}$. This proves the lemma.

\section{PROOF OF THEOREM 1}

We assume that there is a non-degenerate system consisting of $s \leq m n$ distinct vector invariants $u_{r}=u_{r_{1}, \ldots, r_{m}}$ algebraically dependent over $k$, and show that this assumption leads to a contradiction.

Let the above system be of the form $U_{s}=\left\{u_{r} \mid r \in \mathcal{M}_{s}\right\}$, where $\mathcal{M}_{s}$ is a subset of the set $R_{m n}$ of cardinality $s$, and let $T_{s}=\left\{t_{r} \mid r \in \mathcal{M}_{s}\right\}$ be the corresponding system of independent variables $t_{r}=t_{r_{1}, \ldots, r_{i m}}$. From our assumption of algebraic dependence of the vector invariants $u_{r}, r \in \mathcal{M}_{s}$ over the field $k$, it follows that there exists a polynomial $F \in k\left[T_{s}\right]$ such that

$$
F\left(U_{s}\right)=0
$$

identically with respect to $x_{11}, \ldots, x_{\mid n}, \ldots, x_{m 1}, \ldots, x_{m n}$. We may assume that $F$ is a polynomial of minimal degree satisfying equality (11). If we differentiate identity (11) with respect to $x_{i j}, 1 \leq i \leq m, 1 \leq j \leq n$, we obtain the system

$$
\sum_{r \in \mathcal{M}_{s}} \frac{\partial F\left(U_{s}\right) \partial u_{r}}{\partial u_{r} \partial x_{i j}}=0, \quad 1 \leq i \leq m, \quad 1 \leq j \leq n,
$$

consisting of $m n$ linear equations, with respect to the $s$ polynomials $\partial F\left(U_{s}\right) / \partial u_{r}$, $r \in \mathcal{M}_{s}$. Since $F$ is a polynomial of minimal degree satisfying relation (11), we have $\partial F\left(U_{s}\right) / \partial u_{r} \neq 0$ at least for one sequence $r=\left(r_{1}, \ldots, r_{m}\right) \in \mathcal{M}_{s}$. Therefore to get the desired contradiction, it is sufficient to establish the existence of a set $\mathcal{N}_{r}=\left\{\left(i_{1}, j_{1}\right), \ldots,\left(i_{s}, j_{s}\right)\right\}$ consisting of $s$ integral pairs $\left(i_{l}, j_{l}\right), 1 \leq l \leq n$, where $1 \leq i_{l} \leq m$, $1 \leq j_{l} \leq n$, for which the determinant

$$
\Delta_{s}=\operatorname{det}\left\|\frac{\partial u_{r}}{\partial x_{i j}}\right\|_{(i, j) \in \mathcal{K}_{r}, r \in \mathcal{M}_{s}}
$$

is not zero.

The existence of such a determinant $\Delta_{s}$ for any $s, 1 \leq s \leq m n$, is provided by Lemma 3 , and this concludes the proof. 


\section{PROOF OF THEOREM 2}

In (2) we set $x_{j}=x_{1 j} z_{1}+\ldots+x_{m j} z_{m}, 1 \leq j \leq n$. We have

$$
\left(x_{1 j} z_{1}+\ldots+x_{m j} z_{m}\right)^{\sigma}=\sum_{\sigma_{1}+\ldots+\sigma_{m}=\sigma} \frac{\sigma !}{\sigma_{1} ! \ldots \sigma_{m} !}\left(\sum_{j=1}^{n} x_{1 j}^{\sigma_{1}} \ldots x_{m j}^{\sigma_{m}}\right) z_{1}^{\sigma_{1}} \ldots z_{m}^{\sigma_{m}},
$$

and then

$$
\sum_{j=1}^{n}\left(x_{1 j} z_{1}+\ldots+x_{m j} z_{m}\right)^{\sigma}=\sum_{\sigma_{1}+\ldots+\sigma_{m}=\sigma} \frac{\sigma !}{\sigma_{1} ! \ldots \sigma_{m} !}\left(\sum_{j=1}^{n} x_{1 j}^{\sigma_{1}} \ldots x_{m j}^{\sigma_{m}}\right) z_{1}^{\sigma_{1}} \ldots z_{m}^{\sigma_{m}} .
$$

On the other hand

$$
\sum_{1 \leq j_{1}<\ldots<j v n} \prod_{s=1}^{v}\left(x_{1 j_{s}} z_{1}+\ldots+x_{m j_{v}} z_{m}\right)=\sum_{r_{1}+\ldots+r_{m}=v} u_{r_{1}, \ldots, r_{m}} z_{1}^{r_{1}} \ldots z_{m}^{r_{m}}
$$

and then, in view of (2),

$$
\begin{aligned}
\sum_{\sigma_{1}+\ldots+\sigma_{m}=\sigma}\left(\sum_{j=1}^{n} x_{1 j}^{\sigma_{1}} \ldots z_{m j}^{\sigma_{m j}}\right) z_{1}^{\sigma_{1}} \ldots z_{m}^{\sigma_{m \prime}} & \\
& =\sum_{l_{1}+2 l_{2}+\ldots+n l_{n}=\sigma} c\left(l_{1}, \ldots, l_{n}\right) \prod_{v=1}^{n}\left(\sum_{r_{1}+\ldots+r_{m}=v} u_{r_{1}, \ldots, r_{m}} z_{1}^{r_{1}} \ldots z_{m}^{r_{i n}}\right)^{l_{v}}
\end{aligned}
$$

Hence

$$
\begin{aligned}
& \sum_{\sigma_{1}+\ldots+\sigma_{m}} \frac{\sigma !}{\sigma_{1} ! \ldots \sigma_{m} !}\left(\sum_{j=1}^{n} x_{1 j}^{\sigma_{1}} \ldots x_{m j}^{\sigma_{m}}\right) z_{1}^{\sigma_{1}} \ldots z_{m}^{\sigma_{m}} \\
& =\sum_{l_{1}+2 l_{2}+\ldots+n l_{n}=\sigma} c\left(l_{1}, \ldots, l_{n}\right) \prod_{v=1}^{n}\left(\sum_{s_{1}+\ldots+s_{m v}=v l_{v}} v_{s_{1 v}, \ldots, s_{m v}} z_{1}^{s_{1}} \ldots z_{m}^{s_{m v}}\right) \\
& =\sum_{l_{1}+2 l_{2}+\ldots+n l_{n}=\sigma} c\left(l_{1}, \ldots, l_{n}\right) \sum_{\sigma_{1}+\ldots+\sigma_{m}=\sigma}\left(\sum_{s_{i 1}+\ldots+s_{i n}=\sigma_{i},} \prod_{1 \leq i \leq m}^{n} v_{v=1} v_{s_{1}, \ldots, s_{m v}}\right) z_{1}^{\sigma_{1}} \ldots z_{m}^{\sigma_{m}} \\
& =\sum_{\sigma_{1}+\ldots+\sigma_{m}=\sigma}\left(\sum_{l_{1}+2 l_{2}+\ldots+n l_{n}=\sigma} c\left(l_{1}, \ldots, c_{n}\right) \sum_{s_{i 1}+\ldots+s_{i n}=\sigma_{i}, 1 \leq i \leq m} \prod_{v=1}^{n} v_{s_{1}, \ldots, s_{w v}}\right) z_{1}^{\sigma_{1}} \ldots z_{m}^{\sigma_{m}}
\end{aligned}
$$

and therefore

$$
\sum_{j=1}^{n} x_{1 j}^{\sigma_{1}} \ldots x_{m j}^{\sigma_{m j}}=\frac{\sigma_{1} ! \ldots \sigma_{m} !}{\sigma !} \sum_{l_{1}+2 l_{2}+\ldots+n l_{n}=\sigma} c\left(l_{1}, \ldots, l_{n}\right) \sum_{s_{i 1}+\ldots+s_{i n}=\sigma_{i}, 1 \leq i \leq m} \prod_{v=1}^{n} v_{s_{1}, \ldots, s_{m v}},
$$

which proves the theorem. 


\section{REFERENCES}

1. N. Bourbaki, Elements of Mathematics, Algebra II. Springer, Berlin, 1990.

2. D. J. Benson, Polynomial Invariants of Finite Groups. Cambridge Univ. Press, Cambridge, 1993.

3. J. A. Dieudonne and J. B. Carrel, Invariant Theory, Old and New. Academic Press, New York, 1971.

4. D. Mumford, Geometric Invariant Theory. Springer, Berlin, 1993.

5. E. Noether, Der Endlichkeitssatz der Invarianten endlicher Gruppen. Math. Ann. (1916) 77, 89-92.

6. T. A. Springer, Invariant Theory. Springer, Berlin, 1977.

7. S. A. Stepanov, Rational exponential sums and L-functions of Artin. Dokl. Soviet Acad. Sci. (1982) 265, 39-42.

8. H. Weyl, The Classical Groups. Princeton Univ. Press, Princeton, 1946. 